Pacific

Journal of

Mathematics

THE GROUP OF ISOMETRIES OF A FINSLER SPACE

Shaoqiang Deng and Zixin Hou 


\title{
THE GROUP OF ISOMETRIES OF A FINSLER SPACE
}

\author{
Shaoqiang Deng And Zixin Hou
}

\begin{abstract}
We prove that the group of isometries of a Finsler space is a Lie transformation group on the original manifold. This generalizes the famous result of Myers and Steenrod on a Riemannian manifold and makes it possible to use Lie theory on the study of Finsler spaces.
\end{abstract}

\section{Introduction.}

Let $(M, F)$ be a Finsler space, where $F$ is positively homogeneous but not necessary absolutely homogeneous. As in the Riemannian case, we have two kinds of definitions of isometry on $(M, F)$. On one hand, we can define an isometry to be a diffeomorphism of $M$ onto itself which preserves the Finsler function. On the other hand, since on $M$ we still have the definition of distance function (although generically it is not a real distance), we can define an ismotry of $(M, F)$ to be a mapping of $M$ onto $M$ which keeps the distance of each pair of points of $M$.

The equivalence of the two definitions of isometry in the Riemannian case is a famous result of Myers and Steenrod. They used this result to prove that the group of isometries of a Riemannian manifold is a Lie transformation groups on the original manifold [5]. This result plays a fundamental role on the theory of homogeneous Riemannian manifolds. Since then, many different proofs were provided, cf., e.g., Palais [6], S. Kobayashi [4].

In this paper we prove that the two definitions of isometry are equivalent for a Finsler space. Then we prove that the group of isometries has a diferentiable structure which turns it into a Lie transformation on the manifold. This result makes it possible to use Lie theory on the study of Finsler spaces.

In this paper, Finsler structure $F$ is only assumed to be positively homogeneous but not necessary absolutely homogeneous. We will not point out this each time. For a mapping $\phi$ of a manifold $M$, we use $d \phi$ to denote its differential. If $p \in M,\left.d \phi\right|_{p}$ will denote the differential of $\phi$ at $p$. The notations of forward and backward metric ball in a Finsler spaces comes from the newly published book by D. Bao, S.S. Chern and Z. Shen [1]. 


\section{A result on distance function.}

Let $(M, F)$ be a Finsler space, $d$ be the distance function of $(M, F)$. We first need to prove a result on the distance function.

Lemma 1.1. Let $x \in M$. Then for any $\epsilon>0$, there exists a neighborhood $U$ of the original of $T_{x}(M)$ such that $\exp _{x}$ is a $C^{1}$-diffeomorphism from $U$ onto its image and for any $A, B \in U, A \neq B$, and any $C^{1}$ curve $\sigma_{0}(s), 0 \leq s \leq 1$, connecting $A$ and $B$ which satisfies $\sigma_{0}(s) \in U$ and $\dot{\sigma}_{0}(s) \neq 0, s \in[0,1]$, we have

$$
\left|\frac{L(\sigma)}{L\left(\sigma_{0}\right)}-1\right| \leq \epsilon
$$

where $L(\cdot)$ denotes the arc length of a curve and $\sigma(s)=\exp _{x} \sigma_{0}(s)$.

Proof. Let $B_{x}(r)=\left\{A \in T_{x}(M) \mid F(x, A)<r\right\}$ be a tangent ball in $T_{x}(M)$ such that $\exp =\exp _{x}$ is a $C^{1}$-diffeomorphism from $B_{x}(r)$ onto $\mathcal{B}_{x}^{+}(x)=\{w \in$ $M \mid d(x, w) \leq r\}$ (cf. [1]). Assume $A, B \in B_{x}(r), A \neq B$. Let $\sigma_{0}(s), 0 \leq s \leq$ 1 be a $C^{1}$ curve connecting $A$ and $B$ and $\forall s, \sigma_{0}(s) \in B_{x}(r)$ and $\dot{\sigma}_{0}(s) \neq 0$. Then we can write the velocity vector of $\sigma_{0}(s)$ as $\dot{\sigma}_{0}(s)=t(s) X(s)$, where $X(s)$ satisfies $F(x, X(s))=\frac{r}{2}, \forall s$, and $t(s) \geq 0$ is a continuous function on $[0,1]$. Therefore the arc length of $\sigma_{0}$ is

$$
L\left(\sigma_{0}\right)=\int_{0}^{1} t(s) F(x, X(s)) d s .
$$

Denote $X_{1}(s)=\left.d\left(\exp _{x}\right)\right|_{\sigma_{0}(s)} X(s)$. Then the velocity vector of the curve $\sigma(s)=\exp _{x}\left(\sigma_{0}(s)\right), 0 \leq s \leq 1$ is

$$
\dot{\sigma}(s)=\left.d\left(\exp _{x}\right)\right|_{\sigma_{0}(s)}(t(s) X(s))=\left.t(s) d\left(\exp _{x}\right)\right|_{\sigma_{0}(s)}(X(s))=t(s) X_{1}(s) .
$$

Therefore, the arc length of $\sigma$ is

$$
L(\sigma)=\int_{0}^{1} t(s) F\left(\sigma(s), X_{1}(s)\right) d s .
$$

Now we select a neighborhood $V_{1}$ of $x$ in $M$ with compact closure which is contained in $\mathcal{B}_{x}^{+}(r)$ and fix a coordinate system $\left(x_{1}, x_{2}, \ldots, x_{n}\right)$ in $V_{1}$. Let $U_{1}=\exp ^{-1} V_{1}$. Suppose $\sigma_{0} \subset U_{1}$. Denote by $M(s)$ the matrix of $\left.d\left(\exp _{x}\right)\right|_{\sigma_{0}(s)}$ under the base $\frac{\partial}{\partial x_{1}}, \frac{\partial}{\partial x_{2}}, \ldots, \frac{\partial}{\partial x_{n}}$. Given any positive number $\delta<\frac{r}{2}$. Since $\left.d\left(\exp _{x}\right)\right|_{0}=I_{n}$ and $\exp$ is $C^{1}$ smooth, there exists a neighborhood $U_{2} \subset U_{1}$ of the original of $T_{x}(M)$ such that for any $C^{1}$ curve $\sigma_{0}$ satisfying $\sigma_{0}(s) \in U_{2}, \forall s$, we have

$$
\|M(s)-I\|<\frac{\delta}{n}, \quad 0 \leq s \leq 1,
$$


where $\|\cdot\|$ denote the maximum of the absolute value of the entries of a matrix. Write $X(s)$ and $X_{1}(s)$ as:

$$
\begin{aligned}
X(s) & =\left.\sum_{j=1}^{n} y_{j}(s) \frac{\partial}{\partial x_{j}}\right|_{x} ; \\
X_{1}(s) & =\left.\sum_{j=1}^{n} y_{j}^{\prime}(s) \frac{\partial}{\partial x_{j}}\right|_{\sigma(s)} .
\end{aligned}
$$

Then we have

$$
\left|y_{j}^{\prime}(s)-y_{j}(s)\right|<\delta, \quad 1 \leq j \leq n \text {. }
$$

Consider the set

$$
\begin{aligned}
C_{0}=\left\{\left(w,\left.\left(d\left(\exp _{x}\right)\right)\right|_{W}\right) y \mid\right. & w \\
y & \left.\in V_{1}, W=T_{W}(T x(M))=T x(M), F(x, y)=\frac{r}{2}\right\} .
\end{aligned}
$$

Since exp is $C^{1}$ smooth, the closure of $C_{0}$ is compact. Hence the Finsler function $F$ is bounded on $C_{0}$. Suppose $F<r_{1}$ on $C_{0}, r_{1}>0$. Now write the Finsler function $F(w, y)$ as $F\left(w, y_{1}, y_{2}, \ldots, y_{n}\right)$ for $y=\left.\sum_{j=1}^{n} y_{j} \frac{\partial}{\partial x_{j}}\right|_{w}$. Consider the closure $D_{1}$ of the set $D_{0}=\left\{(w, y) \in T M \mid w \in V_{1}, F(x, y) \leq \frac{r}{2}+r_{1}\right\}$. Since $F$ is continuous and $D_{1}$ is compact, $F$ is uniformly continuous on $D_{1}$. Therefore for the given $\epsilon>0$, there exists $\delta_{1}>0$ and a neighborhood $V_{2} \subset V_{1}$ of $x$ such that for any $w \in V_{2},\left|y_{j}-y_{j}^{\prime}\right|<\delta_{1}, j=1,2, \ldots, n$, $F\left(x, y_{1}, y_{2}, \ldots, y_{n}\right)<\frac{r}{2}+r_{1}, F\left(w, y_{1}^{\prime}, \ldots, y_{n}^{\prime}\right)<\frac{r}{2}+r_{1}$, we have

$$
\left|F\left(x, y_{1}, y_{2}, \ldots, y_{n}\right)-F\left(w, y_{1}^{\prime}, y^{\prime} 2, \ldots, y_{n}^{\prime}\right)\right|<\frac{r}{2} \epsilon .
$$

Therefore if we select the above $\delta$ such that $\delta<\delta_{1}$. Then for the corresponding $U_{2}$ and any $C^{1}$ curve $\sigma_{0}, \sigma_{0} \subset U_{2} \cap(\exp )^{-1} V_{2}$, we have

$$
\begin{aligned}
\left|\frac{L(\sigma)}{L\left(\sigma_{0}\right)}-1\right| & =\frac{\left|\int_{0}^{1} t(s)\left(F(x, X(s))-F\left(\sigma(s), X_{1}(s)\right)\right) d s\right|}{\left|\int_{0}^{1} t(s) F(x, X(s)) d s\right|} \\
& \leq \frac{\int_{0}^{1} t(s)\left|F(x, X(s))-F\left(\sigma(s), X_{1}(s)\right)\right| d s}{r \int_{0}^{1} t(s) d s} \\
& \leq \frac{\frac{r}{2} \epsilon \int_{0}^{1} t(s) d s}{\frac{r}{2}} \frac{\int_{0}^{1} t(s) d s}{\int_{0}^{1}} .
\end{aligned}
$$


Theorem 1.2. Let $x \in M$ and $B_{x}(r)$ be a tangent ball of $T_{x}(M)$ such that $\exp _{x}$ is a $C^{1}$ diffeomorphism from $B_{x}(r)$ onto $\mathcal{B}_{x}^{+}(r)$. For $A, B \in B_{x}(r)$, $A \neq B$, let $a=\exp _{x} A, b=\exp _{x} B$. Then we have

$$
\frac{F(x, A-B)}{d(a, b)} \rightarrow 1
$$

as $(A, B) \rightarrow(0,0)$.

Proof. Let $\mathcal{B}_{x}^{-}(r)=\{w \in M \mid d(w, x)<r\}$. Suppose $r$ is so small that each pair of points in $\mathcal{B}_{x}^{+}\left(\frac{r}{2}\right) \cap \mathcal{B}_{x}^{-}\left(\frac{r}{2}\right)$ can be joint by a unique minimal geodesic contained in $\mathcal{B}_{x}^{+}(r)$ (cf. [1]). Let $\Gamma_{0}(s), 0 \leq s \leq 1$ be the line segment connecting $A$ and $B$, and $\Gamma(s)=\exp _{x} \Gamma_{0}(s)$. By Lemma 1.1, we have

$$
\frac{L\left(\Gamma_{0}\right)}{L(\Gamma)}=\frac{F(x, A-B)}{L(\Gamma)} \rightarrow 1
$$

as $(A, B) \rightarrow(0,0)$. Now let $a=\exp _{x} A, b=\exp _{x} B$. Suppose $a, b \in \mathcal{B}_{x}^{+}\left(\frac{r}{2}\right) \cap$ $\mathcal{B}_{x}^{-}\left(\frac{r}{2}\right)$. Let $\gamma_{a b}(s), 0 \leq s \leq 1$ be the unique minimal geodesic of constant speed connecting $a$ and $b$. Let $\gamma_{0}(s), 0 \leq s \leq 1$ be the unique curve in $B_{x}(r)$ which satisfies $\gamma_{a b}(s)=\exp _{x} \gamma_{0}(s)$. Then by Lemma 1.1, we also have

$$
\frac{L\left(\gamma_{0}\right)}{L\left(\gamma_{a b}\right)} \rightarrow 1
$$

as $(A, B) \rightarrow(0,0)$. Since

$$
d(a, b) \leq L(\Gamma), L\left(\gamma_{0}\right) \geq F(x, A-B),
$$

we have

$$
\frac{F(x, A-B)}{L(\Gamma)} \leq \frac{F(x, A-B)}{d(a, b)} \leq \frac{L\left(\gamma_{0}\right)}{L\left(\gamma_{a b}\right)} .
$$

Theorem 1.2 follows.

\section{Differentiability of isometries.}

First we have:

Proposition 2.1. Let $\|\cdot\|_{1},\|\cdot\|_{2}$ be two Minkowski norms on $\mathbb{R}^{n}$. Let $\phi$ be a mapping of $\mathbb{R}^{n}$ into itself such that $\|\phi(A)-\phi(B)\|_{2}=\|A-B\|_{1}$, $\forall A, B \in \mathbb{R}^{n}$. Then $\phi$ is a diffeomorphism.

Proof. Consider $\mathbb{R}^{n}$ endowed with $\|\cdot\|_{j}, j=1,2$ as two Finsler spaces, denoted by $\left(M_{1}, F_{1}\right),\left(M_{2}, F_{2}\right)$. Then geodesics in $M_{j}, j=1,2$ are straight lines (cf. [1]). And the distance function of $M_{j}$ are $d_{j}(A, B)=\|A-B\|_{j}, j=1,2$. Consider $\phi$ as a mapping from the Finsler space $\left(M_{1}, F_{1}\right)$ to $\left(M_{2}, F_{2}\right)$. Then $\phi$ preserves the distance function. Since in a Finsler space short geodesics minimize distance between its start and end points (cf. [1]), we can prove (similarly as in the Riemannian case) that $\phi$ transforms geodesics to geodesics. First suppose $\phi(0)=0$. For $A \in \mathbb{R}^{n}, A \neq 0$, the curve $\phi(t A)$, 
$t \geq 0$ is a ray which coincides with the ray $t \phi(A)$ for $t=0$ and $t=1$. Therefore they coincide as point sets. Thus $\phi(t A)=\mu(t) \phi(A)$ for some nonnegative function $\mu(t)$. Since

$$
\begin{aligned}
\|\phi(t A)-0\|_{2} & =\|t A-0\|_{1}=t\|A\|_{1} \\
& =\|\mu(t) \phi(A)-0\|_{2}=\mu(t)\|\phi(A)\|_{2}=\mu(t)\|A\|_{1}, t \geq 0,
\end{aligned}
$$

we have $\mu(t)=t$. Thus $\phi(t A)=t \phi(A)$, for $t \geq 0$. Suppose $A \neq B$, a similar argument as the above shows that there exists a nonnegative function $\lambda(t)$ such that $\phi(t A+(1-t) B)=\lambda(t) \phi(A)+(1-\lambda(t)) \phi(B), t \geq 0$. And we can similarly show that $\lambda(t)=t$. In particular, for $t=\frac{1}{2}$ we have,

$$
\frac{1}{2} \phi(A+B)=\phi\left(\frac{1}{2}(A+B)\right)=\frac{1}{2} \phi(A)+\frac{1}{2} \phi(B) .
$$

Thus $\phi(A+B)=\phi(A)+\phi(B)$. Taking $A=-B$ in the above equality we have $\phi(-A)=-\phi(A)$. Therefore $\phi$ is a linear transformation. Since $\operatorname{Ker}(\phi)=$ $\{0\}$, it is a diffeomorphism. If $A_{1}=\phi(0) \neq 0$, consider the composition mapping $\phi_{1}=\pi_{A_{1}} \circ \phi$, where $\pi_{A_{1}}(A)=A-A_{1}$ is the parallel translation, which is a diffeomorphism. Since $\phi_{1}(0)=0$ and $\left\|\phi_{1}(A)-\phi(B)\right\|_{2}=\|A-B\|_{1}$, $\phi_{1}$ is a diffeomorphism. Hence $\phi$ is a diffeomorphism.

Remark. The proposition is an interesting application of Finsler geometry to Functional Analysis.

Now we can prove the main result of this paper.

Theorem 2.2. Let $(M, F)$ be a Finsler space and $\phi$ be a distance-preserving mapping of $M$ onto itself. Then $\phi$ is a diffeomorphism.

Proof. Let $p \in M$ and put $q=\phi(p)$. Let $r>0, \epsilon>0$ be so small that both $\exp _{p}$ and $\exp _{q}$ are $C^{1}$ diffeomorphisms on the tangent ball $B_{p}(r+\epsilon), B_{q}(r+\epsilon)$ of $T_{p}(M)$ and $T_{q}(M)$, respectively. For any nonzero $X \in T_{p}(M)$, consider the radial geodesic $\exp _{p}(t X), 0 \leq t \leq \frac{r}{2 F(p, X)}$. The image $\gamma(t)=\phi\left(\exp _{p}(t X)\right)$ is a geodesic since $\phi$ is distance-preserving. Let $X^{\prime}$ denote the tangent vector of $\gamma$ at the point $q$. We have obtained a mapping $X \rightarrow X^{\prime}$ of $T_{p}(M)$ into $T_{q}(M)$. Denoting this mapping by $\phi^{\prime}$ we have $\phi^{\prime}(\lambda X)=\lambda \phi^{\prime}(X)$, for $X \in T_{p}(M)$ and $\lambda \geq 0$. Let $A, B \in T_{p}(M), A \neq B$ and $t$ is so small that both $t A$ and $t B$ lie in $B_{p}(r)$. Let $a_{t}=\exp _{p}(t A), b_{t}=\exp _{p}(t B)$. Then by Theorem 1.2 we have

$$
\lim _{t \rightarrow 0^{+}} \frac{F(p, t A-t B)}{d\left(a_{t}, b_{t}\right)}=1 .
$$

On the other hand, by the definition of $\phi^{\prime}$ we have

$$
\exp _{q}\left(\phi^{\prime}(t X)\right)=\phi(\exp \mathrm{tX})
$$


for any $X$ and $t$ small enough. Thus by Theorem 1.2 we also have

$$
\lim _{t \rightarrow 0^{+}} \frac{F\left(q, \phi^{\prime}(t A)-\phi^{\prime}(t B)\right)}{d\left(\phi\left(a_{t}\right), \phi\left(b_{t}\right)\right)}=1 .
$$

Since $d\left(\phi\left(a_{t}\right), \phi\left(b_{t}\right)\right)=d\left(a_{t}, b_{t}\right)$, we get

$$
\begin{aligned}
1 & =\lim _{t \rightarrow 0^{+}} \frac{F(p, t A-t B)}{F\left(q, \phi^{\prime}(t A)-\phi^{\prime}(t B)\right)} \\
& =\lim _{t \rightarrow 0^{+}} \frac{t F(p, A-B)}{t F\left(q, \phi^{\prime}(A)-\phi^{\prime}(B)\right)}=\frac{F(p, A-B)}{F\left(q, \phi^{\prime}(A)-\phi^{\prime}(B)\right)} .
\end{aligned}
$$

Therefore $F\left(q, \phi^{\prime}(A)-\phi^{\prime}(B)\right)=F(p, A-B)$. By Proposition 2.1, $\phi^{\prime}$ is a diffeomorphism of $T_{p}(M)$ onto $T_{q}(M)$.

Although on $\mathcal{B}_{p}^{+}(r)=\exp _{p} B_{r}(p)$ we have $\phi=\exp _{q} \circ \phi^{\prime} \circ\left(\exp _{p}\right)^{-1}$, we still cannot conclude that $\phi$ is smooth on $\mathcal{B}_{p}^{+}(r)$, since in a Finsler space the exponential mapping is only $C^{1}$ at the zero section. That is, we can only conclude that $\phi$ is smooth in $\mathcal{B}_{p}(r)-\{p\}$. To finish the proof, we proceed to take $r$ so small so that every pair of points in $\mathcal{B}_{p}^{+}(r) \cap \mathcal{B}_{p}^{-}(r)$ can be joint by a unique minimizing geodesic. Select $p_{1} \in \mathcal{B}_{p}^{+}\left(\frac{r}{2}\right) \cap \mathcal{B}_{p}^{-}\left(\frac{r}{2}\right), p_{1} \neq p$. Consider the tangent ball $B_{p_{1}}\left(\frac{r}{2}\right)$ of $T_{p_{1}}(M)$. The exponential mapping is a $C^{1}$ diffeomorphism from $B_{p_{1}}\left(\frac{r}{2}\right)$ onto $\mathcal{B}_{p_{1}}^{+}\left(\frac{r}{2}\right)$. The above argument shows that $\phi$ is smooth in $\mathcal{B}_{p_{1}}^{+}\left(\frac{r}{2}\right)-\left\{p_{1}\right\}$, which is a neighborhood of $p$. This completes the proof.

\section{Group of isometries.}

Theorem 2.2 justifies the following definition of isometry for a Finsler space.

Definition 3.1. Let $(M, F)$ be a Finsler space. A mapping $\phi$ of $M$ onto itself is called an isometry if $\phi$ is a diffeomorphism and for any $x \in M, X \in$ $T_{x}(M), F\left(\phi(x), d \phi_{x}(X)\right)=F(x, X)$.

In the following we denote the group of isometries of $(M, F)$ by $I(M)$.

Let $N$ be a connected, locally compact metric space and $\mathcal{I}(N)$ be the group of isometries of $N$, for each point $x$ of $N$, let $\mathcal{I}_{x}(N)$ denote the isotropy subgroup of $\mathfrak{I}(N)$ at $x$. Van Danzig and van der Waerden [7] proved that $\mathcal{I}(N)$ is a locally compact topological transformation group on $N$ with respect to the compact-open topology and $\mathcal{I}_{x}(N)$ is compact.

Now on $M$ we have a distance function $d$ defined by the Finsler function $F$. By Theorem 2.2, the group $I(M)$ coincides with the group of isometries $\mathcal{I}(M)$ of $(M, d)$. Although generically $d$ is not a distance ( $d$ is not symmetric unless $F$ is absolutely homogeneous), we still have:

Theorem 3.2. Let $(M, F)$ be a connected Finsler space. The compact-open topology turns $I(M)$ into a locally compact transformation group of $M$. Let 
$x \in M$ and $I_{x}(M)$ denote the subgroup of $I(M)$ which leaves $x$ fixed. Then $I_{x}(M)$ is compact.

Proof. A proof of this result for the Riemannian case was given in Helgason [3] (cf. Helgason [3], pp. 201-204), which is valid in general cases after some minor changes. Just note that on a Finsler manifold the topology generated by the forward metric balls $\mathcal{B}_{p}^{+}(r)=\{x \in M \mid d(p, x)<r\}, p \in M, r>0$ is precisely the underlying manifold topology and this is true for the topology generated by the backward metric balls $\mathcal{B}_{p}^{-}(r)=\{x \in M \mid d(x, p)<r\}, p \in$ $M, r>0$ (cf. [1] ).

Bochner-Montgomery [2] proved that a locally compact group of differentiable transformations of a manifold is a Lie transformation group. Therefore we have the following theorem.

Theorem 3.3. Let $(M, F)$ be a Finsler space. Then the group of isometries $I(M)$ of $M$ is a Lie transformation group of $M$. Let $x \in M$ and $I_{x}(M)$ be the isotropy subgroup of $I(M)$ at $x$. Then $I_{x}(M)$ is compact.

\section{References}

[1] D. Bao, S.S. Chern and Z. Shen, An Introduction to Riemannian-Finsler Geometry, Springer-Verlag, Berlin, 1999, MR 2001g:53130, Zbl 0954.53001.

[2] S. Bochner and D. Montgomery, Locally compact groups of differentiable transformations, Ann. of Math., 47 (1946), 639-653, MR 8,253c, Zbl 0061.04407.

[3] S. Helgason, Differential Geometry, Lie Groups and Symmetric Spaces, 2nd ed., Academic Press, New York, 1978, MR 80k:53081, Zbl 0451.53038.

[4] S. Kobayashi, Transformation Groups in Differential Geometry, Springer-Verlag, Berlin, 1972, MR 50 \#8360, Zbl 0246.53031.

[5] S.B. Myers and N. Steenrod, The group of isometries of a Riemannian manifold, Ann. of Math., 40 (1939), 400-416, Zbl 0021.06303.

[6] R. Palais, On the differentiability of isometries, Proc. Math. AMS., 8 (1957), 805-807, MR 19,451a, Zbl 0084.37405.

[7] D. van Dantzig and B.L. van der Waerden, Über metriseh homogene räume, Abh. Math. Sem. Univ. Hamburg, 6 (1928), 374-376.

Received March 15, 2001. This work is Project 19901015 and 19731004 supported by NSFC.

Department of Mathematics

NANKAI UNIVERSITY

Tianjin, P. R. China

E-mail address: matdsq@263.net

Department of Mathematics

NANKAI UNIVERSITY

Tianjin, P. R. China

E-mail address: houzx@nankai.edu.cn 